\title{
ON INTEGRATION OF THE LOADED KORTEWEG-DE VRIES EQUATION IN THE CLASS OF RAPIDLY DECREASING FUNCTIONS
}

\author{
AKNAZAR B. HASANOV AND UMID A. HOITMETOV
}

\begin{abstract}
In this work, the inverse scattering method is applied to the integration of the loaded Korteweg-de Vries equation in the class of rapidly decreasing functions. The multisoliton solutions of the loaded Korteweg-de Vries equation are found.
\end{abstract}

\section{Introduction}

In this paper, we study the loaded Korteweg-de Vries (KdV) equation, namely, we consider the following equation:

$$
u_{t}+\beta(t) u\left(x_{0}, t\right)\left(u_{x x x}-6 u u_{x}\right)+\gamma(t) u\left(x_{1}, t\right) u_{x}=0,
$$

where $\beta(t)$ and $\gamma(t)$ are given continuously differentiable functions and $x_{0}, x_{1} \in \mathbb{R}$. Equation (1.1) is considered with the initial condition

$$
u(x, 0)=u_{0}(x), \quad x \in \mathbb{R},
$$

where the initial function $u_{0}(x)$ has the following properties:

1)

$$
\int_{-\infty}^{\infty}(1+|x|)\left|u_{0}(x)\right| d x<\infty
$$

2) the operator $L(0):=-\frac{d^{2}}{d x^{2}}+u_{0}(x), x \in \mathbb{R}$ has equally $N$ negative eigenvalues $\lambda_{1}(0), \lambda_{2}(0), \ldots, \lambda_{N}(0)$.

It is required to find a function $u(x, t)$ that is sufficiently smooth and tends to its limits rather quickly at $x \rightarrow \pm \infty$, incl.

$$
\int_{-\infty}^{\infty}\left((1+|x|)|u(x, t)|+\left|\frac{\partial^{j} u(x, t)}{\partial x^{j}}\right|\right) d x<\infty, \quad j=1,2,3 .
$$

The purpose of this work is to develop an algorithm for constructing the solution $u(x, t)$ to problem (1.1) - (1.4) using the inverse scattering problem method for the self-adjoint Sturm-Liouville operator.

2010 Mathematics Subject Classification. 34L25, 35P25, 47A40, 37K15.

Key words and phrases. Loaded Korteweg-de Vries equation, Sturm-Liouville operator, Jost solutions, inverse scattering theory, Gelfand-Levitan-Marchenko integral equation, evolution of the scattering data, soliton solutions. 
The inverse scattering method dates back to the work of Gardner, Greene, Kruskal, and Miura [5]. They succeeded in finding a global solution to the Cauchy problem for the KdV equation by reducing it to the inverse scattering problem for the self-adjoint Sturm-Liouville operator. This inverse scattering problem was first solved in the work of L.D. Faddeev [3], then in the works of V.A. Marchenko [18], B.M. Levitan [15], and others. In [13] P. Lax showed the universality of the inverse scattering method and generalized the $\mathrm{KdV}$ equation by introducing the concept of the higher KdV equation.

Interest in solitons was renewed in connection with research in plasma physics. In 1958, R.Z. Sagdeev [22] showed that solitons can propagate in plasma, which are quite similar to solitons on the surface of a liquid. Gardner and Morikawa [6] established a direct analogy between the equations describing plasma in a strong magnetic field and the equations of shallow water. From that time on, the KdV equation acquired a general physical character, and soon the possibility of its application to various wave problems was established. Problems in the theory of solitons have been considered in many monographs and articles, of which [1, 2, 23, 27] should be noted.

In recent years, in connection with the intensive study of the problems of optimal management of the agroecosystem for example, the problem of long-term forecasting and regulation of the level of groundwater and soil moisture, interest in loaded equations has significantly increased. Loaded differential equations in the literature are usually called equations containing in the coefficients or in the right-hand side any functionals of the solution, in particular, the values of the solution or its derivatives on manifolds of lower dimension. The study of such equations is of interest both from the point of view of constructing a general theory of differential equations and from that of applications. Among the works devoted to loaded equations, the works by A.M.Nakhushev [20, 21], I.A.Kozhanov $[12]$ and others should be specially mentioned.

Equations of the form (1.1) without a loaded term are also encountered in applied mechanics. For example, in the works of A.A. Lugovtsov [16, 17], the system of equations describing the propagation of one-dimensional nonlinear waves in an inhomogeneous gas-liquid medium is reduced to one equation of the form

$$
u_{\tau}+\alpha(\tau) u u_{\eta}+\beta(\tau) u_{\eta \eta \eta}-\mu(\tau) u_{\eta \eta}+\left[\frac{k}{2 \tau}+\delta(\tau)\right] u=0 .
$$

In particular, for $\mu=0, k=1, \delta=0$ it is shown that under certain conditions cylindrical waves can exist in the form of solitons.

Note that the KdV equation and the general KdV equation with self-consistent sources in the class of rapidly decreasing and step-like functions were studied in $[8,9,11,14,19,24]$ and the integration of the loaded $\mathrm{KdV}$ equation in the class of periodic functions was investigated in $[10,25]$.

\section{Preliminaries}

Consider the Sturm-Liouville equation

$$
L(0) y:=-y^{\prime \prime}+u_{0}(x) y=k^{2} y, \quad x \in \mathbb{R},
$$


where the potential $u_{0}(x)$ satisfies condition (1.3). In this subsection, we provide the information necessary for the further presentation concerning the direct and inverse scattering problems for equation (2.1).

We denote by $f(x, k)$ and $g(x, k)$ the Jost solutions of Eq. (2.1) with the asymptotics

$$
\begin{gathered}
f(x, k)=e^{i k x}+o(1), \quad x \rightarrow \infty, \quad(\operatorname{Im} k=0) ; \\
g(x, k)=e^{-i k x}+o(1), \quad x \rightarrow-\infty, \quad(\operatorname{Im} k=0) .
\end{gathered}
$$

Under condition (1.3), such solutions exist and are uniquely determined by asymptotics (2.2).

The solutions $f(x, k), g(x, k)$ satisfy the representations

$$
\begin{gathered}
f(x, k)=e^{i k x}+\int_{x}^{\infty} K^{+}(x, z) e^{i k z} d z, \\
g(x, k)=e^{-i k x}+\int_{-\infty}^{x} K^{-}(x, z) e^{-i k z} d z,
\end{gathered}
$$

where kernels $K^{+}(x, z), K^{-}(x, z)$ are real functions related to the potential $u_{0}(x)$ by the relations

$$
u_{0}(x)=\mp 2 \frac{d}{d x} K^{ \pm}(x, x) .
$$

With respect to the variable $k$, the Jost solutions $f(x, k)$ and $g(x, k)$ continue analytically into the upper half-plane $\operatorname{Im} k>0$.

For real $k$, pairs of functions $\{f(x, k), f(x,-k)\}$ and $\{g(x, k), g(x,-k)\}$ are pairs of linearly independent solutions of equation (2.1), therefore

$$
\begin{gathered}
f(x, k)=b(k) g(x, k)+a(k) g(x,-k), \\
g(x, k)=-b(-k) f(x, k)+a(k) f(x,-k) .
\end{gathered}
$$

The functions $r^{+}(k)=-\frac{b(-k)}{a(k)}$ and $r^{-}(k)=\frac{b(k)}{a(k)}$ are called reflection coefficients (right and left, respectively). The coefficients $a(k), b(k)$ and $r^{+}(k)$ have the following properties (see [15], p.121):

A. For real $k \neq 0$

$$
\begin{gathered}
a(-k)=\overline{a(k)}, \quad b(-k)=\overline{b(k)}, \quad|a(k)|^{2}=1+|b(k)|^{2} \\
a(k)=-\frac{1}{2 i k} W\{f(x, k), g(x, k)\}, \quad b(k)=\frac{1}{2 i k} W\{f(x, k), g(x,-k)\} \\
a(k)=1+O\left(\frac{1}{k}\right), b(k)=O\left(\frac{1}{k}\right), \quad k \rightarrow \pm \infty ;
\end{gathered}
$$

where

$$
W\{f(x, k), g(x, k)\} \equiv f(x, k) g^{\prime}(x, k)-f^{\prime}(x, k) g(x, k) ;
$$

B. The function $a(k)$ extends analytically to the half-plane $\operatorname{Im} k>0$ and there it has a finite number of zeros $k_{n}=i \chi_{n},\left(\chi_{n}>0\right), n=1,2, \ldots, N$, these zeros are simple, and $\lambda_{n}=-\chi_{n}^{2}, n=1,2, \ldots, N$ are the eigenvalues of the operator $L(0)$. In addition, following relation holds

$$
g\left(x, i \chi_{j}\right)=B_{j} f\left(x, i \chi_{j}\right), \quad j=1,2, \ldots, N
$$


C. For real $k \neq 0$, the function $r^{+}(k)$ is continuous,

$$
\begin{gathered}
\overline{r^{+}(k)}=r^{+}(-k), \quad\left|r^{+}(k)\right|<1, \quad r^{+}(k)=o\left(k^{-1}\right), \quad|k| \rightarrow \infty, \\
k^{2}\left[1-\left|r^{+}(k)\right|^{2}\right]^{-1}=O(1), \quad|k| \rightarrow 0 ;
\end{gathered}
$$

D. Function $k(a(k)-1)$, where $a(k)$ is defined by the formula

$$
a(k)=\prod_{j=1}^{N} \frac{k-i \chi_{j}}{k+i \chi_{j}} \exp \left\{-\frac{1}{2 \pi i} \int_{-\infty}^{\infty} \frac{\ln \left(1-\left|r^{+}(\xi)\right|^{2}\right)}{\xi-k} d \xi\right\}, \quad \operatorname{Im} k>0,
$$

continuous and bounded in $\operatorname{Im} k \geq 0$ and

$$
\begin{aligned}
& (a(k))^{-1}=O(1), \quad|k| \rightarrow 0, \quad \operatorname{Im} k \geq 0, \\
& \lim _{k \rightarrow 0} k a(k)\left(r^{+}(k)+1\right)=0, \quad \operatorname{Im} k=0 ;
\end{aligned}
$$

E. Functions

$$
R^{ \pm}(x)=\frac{1}{2 \pi} \int_{-\infty}^{\infty} r^{ \pm}(k) e^{i k x} d k
$$

for each $a>-\infty$ satisfy the condition

$$
(1+|x|)\left|R^{ \pm}( \pm x)\right| \in L^{1}(a, \infty) .
$$

The set $\left\{r^{+}(k), \chi_{1}, \chi_{2}, \ldots, \chi_{N}, B_{1}, B_{2}, \ldots, B_{N}\right\}$ is called the scattering data for problem $(1.3)+(2.1)$. The direct scattering problem is to determine the scattering data from the potential $u_{0}(x)$, and the inverse problem is to reconstruct the potential $u_{0}(x)$ from the scattering data of Eq. (2.1).

The kernel $K^{+}(x, y)$ in representation (2.3) is a solution to the Gelfand-LevitanMarchenko integral equation

$$
K^{+}(x, y)+F^{+}(x+y)+\int_{x}^{\infty} K^{+}(x, z) F^{+}(z+y) d z=0, \quad(y>x),
$$

where

$$
\begin{gathered}
F^{+}(x)=\sum_{j=1}^{N} \alpha_{j}^{+} e^{-\chi_{j} x}+\frac{1}{2 \pi} \int_{-\infty}^{\infty} r^{+}(k) e^{i k x} d k \\
\alpha_{j}^{+}=\frac{B_{j}}{\left.i \frac{d a(z)}{d z}\right|_{z=i \chi_{j}}}
\end{gathered}
$$

and $a(z)$ is the analytic continuation of the function $a(k)$ to the upper half-plane $\operatorname{Im} k>0$.

The following theorem is true $[7,26]$.

Theorem 2.1. Specifying the scattering data uniquely determines the potential $u_{0}(x)$. 


\section{Evolution of scattering data}

Consider the following $\mathrm{KdV}$ equation

$$
u_{t}+\beta(t) u\left(x_{0}, t\right)\left(u_{x x x}-6 u u_{x}\right)=G(x, t),
$$

where

$$
G(x, t)=-\gamma(t) u\left(x_{1}, t\right) u_{x} .
$$

For equation (3.1), we will seek a Lax pair [4] in the form

$$
\begin{gathered}
-\Phi_{x x}+\left(u-k^{2}\right) \Phi=0, \\
\Phi_{t}=\beta(t) u\left(x_{0}, t\right)\left(-u_{x}+4 i k^{3}\right) \Phi+\beta(t) u\left(x_{0}, t\right)\left(2 u+4 k^{2}\right) \Phi_{x}+F(x, t) .
\end{gathered}
$$

Using the identity $\Phi_{x x t}=\Phi_{t x x}$, based on equalities (3.1) - (3.4), we obtain

$$
-F_{x x}+\left(u(x, t)-k^{2}\right) F=-G(x, t) \Phi .
$$

Letting $\Phi(x, t)=g(x, k ; t)$, we seek a solution to Eq. (3.5) in the form

$$
F=B(x) g(x, k ; t)+C(x) g(x,-k ; t) .
$$

Then, to determine $B(x)$ and $C(x)$, we obtain the following system of equations

$$
\begin{gathered}
B^{\prime}(x) g(x, k ; t)+C^{\prime}(x) g(x,-k ; t)=0, \\
B^{\prime}(x) g^{\prime}(x, k ; t)+C^{\prime}(x) g^{\prime}(x,-k ; t)=G(x, t) g(x, k ; t),
\end{gathered}
$$

which has the solution as

$$
\begin{gathered}
B(x)=-\frac{1}{2 i k} \int_{-\infty}^{x} g(s, k ; t) g(s,-k ; t) G(s, t) d s, \\
C(x)=\frac{1}{2 i k} \int_{-\infty}^{x} g^{2}(s, k ; t) G(s, t) d s .
\end{gathered}
$$

Therefore, using expression (3.2), the equation (3.4) can be rewritten as follows

$$
\begin{gathered}
\frac{\partial g(x, k ; t)}{\partial t}=\beta(t) u\left(x_{0}, t\right)\left(-u_{x}+4 i k^{3}\right) g(x, k ; t) \\
+\beta(t) u\left(x_{0}, t\right)\left(2 u+4 k^{2}\right) \frac{\partial g(x, k ; t)}{\partial x} \\
+\frac{\gamma(t) u\left(x_{1}, t\right) g(x, k ; t)}{2 i k} \int_{-\infty}^{x} g(s, k ; t) g(s,-k ; t) u_{s}(s, t) d s \\
-\frac{\gamma(t) u\left(x_{1}, t\right) g(x,-k ; t)}{2 i k} \int_{-\infty}^{x} g^{2}(s, k ; t) u_{s}(s, t) d s .
\end{gathered}
$$

Passing in equality (3.6) to the limit $x \rightarrow \infty$, by virtue of (2.8), (2.7) and the asymptotics of the Jost solution, we derive

$$
\begin{gathered}
\frac{d a(k ; t)}{d t}=\frac{\gamma(t) u\left(x_{1}, t\right) a(k ; t)}{2 i k} \int_{-\infty}^{\infty} g(s, k ; t) g(s,-k ; t) u_{s}(s, t) d s \\
+\frac{\gamma(t) u\left(x_{1}, t\right) b(k ; t)}{2 i k} \int_{-\infty}^{\infty} g^{2}(s, k ; t) u_{s}(s, t) d s \\
\frac{d b(-k ; t)}{d t}=8 i k^{3} \alpha(t) u\left(x_{0}, t\right) b(-k ; t) \\
+\frac{\gamma(t) u\left(x_{1}, t\right) b(-k ; t)}{2 i k} \int_{-\infty}^{\infty} g(s, k ; t) g(s,-k ; t) u_{s}(s, t) d s
\end{gathered}
$$




$$
+\frac{\gamma(t) u\left(x_{1}, t\right) a(-k ; t)}{2 i k} \int_{-\infty}^{\infty} g^{2}(s, k ; t) u_{s}(s, t) d s .
$$

Multiplying (3.8) by $a(k, t)$ and subtracting from it equality (3.7) multiplied by $b(-k, t)$, according to $(2.11)$, we obtain

$$
\frac{d r^{+}(k, t)}{d t}=8 i k^{3} \beta(t) u\left(x_{0}, t\right) r^{+}(k, t)-\frac{\gamma(t) u\left(x_{1}, t\right)}{2 i k a^{2}(k ; t)} \int_{-\infty}^{\infty} g^{2}(s, k ; t) u_{s}(s, t) d s .
$$

Let us calculate the integral on the right-hand side of the previous equality. To do this, we use formula (2.7) and we have

$$
\begin{aligned}
\int_{-\infty}^{\infty} g^{2}(s, k ; t) u_{s}(s, t) d s \\
\quad=-2 \int_{-\infty}^{\infty}\left(g^{\prime \prime}(s, k ; t)+k^{2} g(s, k ; t)\right) g^{\prime}(s, k ; t) d s=4 k^{2} a(k) b(-k) .
\end{aligned}
$$

According to this and equality $(3.7)$, we have $a_{t}(k, t)=0$. Therefore, we deduce that

$$
\begin{gathered}
\frac{d \lambda_{j}(t)}{d t}=0, \\
\frac{d r^{+}(k, t)}{d t}=\left(8 i k^{3} \beta(t) u\left(x_{0}, t\right)-2 i k \gamma(t) u\left(x_{1}, t\right)\right) r^{+}(k, t) .
\end{gathered}
$$

We now turn to find the evolution of the normalizing numbers $B_{n}, n=1,2, \ldots, N$ corresponding to the eigenvalues $\lambda_{n}, n=1,2, \ldots N$. For this, we rewrite equality (3.6) in the following form

$$
\begin{gathered}
\frac{\partial g(x, k ; t)}{\partial t}=\beta(t) u\left(x_{0}, t\right)\left(-u_{x}+4 i k^{3}\right) g(x, k ; t)+\beta(t) u\left(x_{0}, t\right)\left(2 u+4 k^{2}\right) \frac{\partial g(x, k ; t)}{\partial x} \\
+\frac{\gamma(t) u\left(x_{1}, t\right) g(x, k ; t)}{2 i k}[g(x, k ; t) g(x,-k ; t) u(x, t) \\
\left.-\int_{-\infty}^{x} u(s, t)\left(g^{\prime}(s, k ; t) g(s,-k ; t)+g(s, k ; t) g^{\prime}(s,-k ; t)\right) d s\right] \\
-\frac{\gamma(t) u\left(x_{1}, t\right) g(x,-k ; t)}{2 i k}\left[g^{2}(x, k ; t) u(x, t)-\int_{-\infty}^{x} 2 g^{\prime}(s, k ; t) g(s, k ; t) u(s, t) d s\right] \\
=\beta(t) u\left(x_{0}, t\right)\left(-u_{x}+4 i k^{3}\right) g(x, k ; t)+\beta(t) u\left(x_{0}, t\right)\left(2 u+4 k^{2}\right) \frac{\partial g(x, k ; t)}{\partial x} \\
-\gamma(t) u\left(x_{1}, t\right) g^{\prime}(x, k ; t)-i k \gamma(t) u\left(x_{1}, t\right) g(x, k ; t) .
\end{gathered}
$$

Using equality (2.9), setting $k=k_{n}$, taking into account the asymptotics of the Jost solution for $x \rightarrow+\infty$ and equating the coefficients for $e^{-\chi_{n} x}$, we find an analogue of the Gardner-Greene-Kruskal-Miura equations

$$
\begin{gathered}
\frac{d B_{n}(t)}{d t}=\left(8 \chi_{n}^{3} \beta(t) u\left(x_{0}, t\right)+2 \chi_{n} \gamma(t) u\left(x_{1}, t\right)\right) B_{n}(t), \\
n=1,2, \ldots, N .
\end{gathered}
$$

Thus, the following theorem has been proved.

Theorem 3.1. If the function $u(x, t)$ is a solution to the problem (1.1) - (1.4), then the scattering data $\left\{r^{+}(k, t), \lambda_{n}(t), B_{n}(t), n=\overline{1, N}\right\}$ of the operator $L(t)$ with the potential $u(x, t)$ satisfy differential equations (3.9), (3.10) and (3.11). 
Remark 3.1. Consider the kernel of the Gelfand-Levitan-Marchenko integral equation

$$
F^{+}(x, t)=\sum_{j=1}^{N} \alpha_{j}^{+}(t) e^{-\chi_{j} x}+\frac{1}{2 \pi} \int_{-\infty}^{\infty} r^{+}(k, t) e^{i k x} d k
$$

with the scattering data from Theorem 3.1. Then the data $\left\{r^{+}(k, t), \chi_{1}(t), \chi_{2}(t), \ldots\right.$, $\left.\chi_{N}(t), B_{1}(t), B_{2}(t), \ldots, B_{N}(t)\right\}$ satisfy conditions A-E. Therefore, according to Theorem 2.1, the potential $u(x, t)$ in the operator $L(t)$ is uniquely determined.

Remark 3.2. The obtained relations completely determine the evolution of the scattering data for the operator $L(t)$ and thus make it possible to apply the inverse scattering method to solve problem (1.1)-(1.4). Let a function $(1+|x|) u_{0}(x) \in$ $L^{1}(\mathbb{R})$ be given. Then the solution to the problem is found using the following algorithm.

- We solve the direct scattering problem with the initial function $u_{0}(x)$ and obtain the scattering data $\left\{r^{+}(k), \chi_{1}, \chi_{2}, \ldots, \chi_{N}, B_{1}, B_{2}, \ldots, B_{N}\right\}$ for the operator $L(0)$.

- Using the results of the Theorem 3.1, we find the scattering data for $t>0$ :

$$
\left\{r^{+}(k, t), \chi_{1}(t), \chi_{2}(t), \ldots, \chi_{N}(t), B_{1}(t), B_{2}(t), \ldots, B_{N}(t)\right\} .
$$

- Using the method based on the Gelfand-Levitan-Marchenko integral equation, we solve the inverse scattering problem, i.e. find $u(x, t)$ from the scattering data for $t>0$ obtained in the previous step.

Example 3.1. Consider the following problem

$$
\begin{gathered}
u_{t}+\beta(t) u(0, t)\left(u_{x x x}-6 u u_{x}\right)+\gamma(t) u(1, t) u_{x}(x, t)=0, \\
u(x, 0)=-\frac{2}{\operatorname{ch}^{2} x}, \quad x \in \mathbb{R},
\end{gathered}
$$

where

$$
\beta(t)=t^{2}+1+\frac{\sqrt{t^{2}+1}}{8}, \quad \gamma(t)=-\left(\frac{\sqrt{t^{2}+1}+t}{e}+\frac{e}{\sqrt{t^{2}+1}+t}\right)^{2} .
$$

It is easy to find the scattering data for the operator $L(0)$ :

$$
\lambda(0)=\left(i \chi_{1}(0)\right)^{2}=-1 ; r^{+}(k, 0)=0, B_{1}(0)=1 .
$$

By Theorem 3.1, we have

$$
\lambda(t)=\lambda(0)=-1 ; r^{+}(k, t)=0, B_{1}(t)=e^{\mu(t)},
$$

where

$$
\mu(t)=8 \int_{0}^{t} \beta(\tau) u(0, \tau) d \tau+2 \int_{0}^{t} \gamma(\tau) u(1, \tau) d \tau
$$

Substituting these data into the formula (2.11), we find the kernel

$$
F_{+}(x, t)=2 e^{-x+\mu(t)}
$$

integral equation of Gelfand-Levitan-Marchenko. Further, solving the integral equation

$$
K_{+}(x, y ; t)+2 e^{\mu(t)} \cdot e^{-(x+y)}+2 e^{\mu(t)} \cdot e^{-y} \int_{x}^{\infty} K_{+}(x, s ; t) e^{-s} d s=0
$$


we get

$$
K_{+}(x, y ; t)=-\frac{2 e^{\mu(t)} \cdot e^{-(x+y)}}{1+e^{\mu(t)} \cdot e^{-2 x}} .
$$

Whence we find the solution to the Cauchy problem (3.12) - (3.13):

$$
u(x, t)=-2 \frac{d}{d x} K^{+}(x, x, t)=-\frac{2}{\operatorname{ch}^{2}(x-\operatorname{arcsh} t)} .
$$

It is known that the solution of the $\mathrm{KdV}$ equation satisfying the initial condition (3.13) looks as follows:

$$
u(x, t)=-\frac{2}{\operatorname{ch}^{2}(x-4 t)} .
$$

The difference between these two solutions is shown in the following figures:
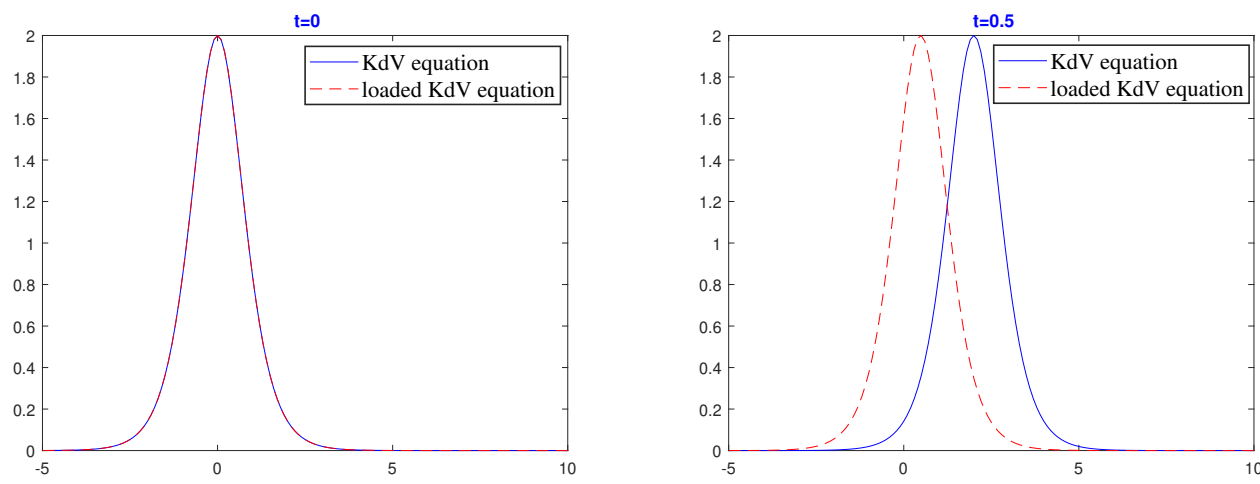

\section{Multisoliton solutions}

One of the remarkable properties of nonlinear evolution equations that are integrable by the inverse problem method is the existence of multisoliton solutions. A remarkable feature of these solutions is that they can be integrated as solutions describing the interaction of $N$ solitons, which after interaction do not change their shape, but only undergo a certain phase shift.

We know that if the function $u(x, t)$ is a solution to problem (1.1) - (1.4), then

$$
\begin{gathered}
\lambda_{n}(t)=\lambda_{n}(0), \\
r^{+}(k, t)=r^{+}(k, 0) \exp \left\{\int_{0}^{t}\left(8 i k^{3} \beta(\tau) u\left(x_{0}, \tau\right)-2 i k \gamma(\tau) u\left(x_{1}, \tau\right)\right) d \tau\right\}, \\
\alpha_{n}^{+}(t)=\alpha_{n}^{+}(0) \exp \left\{\int_{0}^{t}\left(8 \chi_{n}^{3} \beta(\tau) u\left(x_{0}, \tau\right)+2 \chi_{n} \gamma(\tau) u\left(x_{1}, \tau\right)\right) d \tau\right\}, \quad n=\overline{1, N} .
\end{gathered}
$$

Let us now consider the case without reflective potentials: $r^{+}(k) \equiv 0$. In this case, the Gelfand-Levitan-Marchenko integral equation is solved explicitly. Suppose all $k_{j}: a\left(k_{j}\right)=0$ are on the imaginary axis, so $k_{j}=i \chi_{j}, 0<\chi_{1}<\chi_{2}<\ldots<\chi_{N}$. Then equation (2.10) can be rewritten as

$$
K^{+}(x, y, t)+\sum_{j=1}^{N} \alpha_{j}^{+}(t) e^{-\chi_{j}(x+y)}+\sum_{j=1}^{N} \alpha_{j}^{+}(t) e^{-\chi_{j} y} \int_{x}^{\infty} K^{+}(x, z) e^{-\chi_{j} z} d z=0
$$


or

$$
K^{+}(x, y, t)+\sum_{j=1}^{N} \alpha_{j}^{+}(t) e^{-\chi_{j} y}\left(e^{-\chi_{j} x}+\int_{x}^{\infty} K^{+}(x, z) e^{-\chi_{j} z} d z\right)=0 .
$$

We put

$$
K^{+}(x, y, t)=\sum_{n=1}^{N} K_{n}(x, t) e^{-\chi_{n} y} .
$$

This gives linear algebraic equations for $K_{n}$ :

$$
\sum_{m=1}^{N}\left(\delta_{n m}+\alpha_{n}^{+}(t) \frac{e^{-\left(\chi_{n}+\chi_{m}\right) x}}{\chi_{n}+\chi_{m}}\right) K_{m}(x, t)=-\alpha_{n}^{+}(t) e^{-\chi_{n} x}, n=1,2, \ldots, N,
$$

where $\delta_{m n}$ is the Kronecker symbol. We denote by $D$ the determinant of the main matrix of the system of linear algebraic equations (4.1):

$$
D=\operatorname{det}\left(\delta_{n m}+\alpha_{n}^{+}(t) \frac{e^{-\left(\chi_{n}+\chi_{m}\right) x}}{\chi_{n}+\chi_{m}}\right) \equiv \operatorname{det}\left(D_{n m}\right) .
$$

Then the solution to the system (4.1) can be written in the form

$$
K_{n}(x, t)=\frac{\operatorname{det} D^{(n)}}{D},
$$

where $D^{(n)}$ is the matrix obtained from $D_{n m}$ by replacing the $n$th column with the column that forms the right-hand side of (4.1). It's obvious that

$$
\frac{d D}{d x}=\sum_{n=1}^{N} e^{-\chi_{n} x} \operatorname{det} D^{(n)}
$$

From (4.2) - (4.4) it follows

$$
K^{+}(x, x, t)=\frac{1}{D} \sum_{n=1}^{N} \operatorname{det} D^{(n)} e^{-\chi_{n} x}=\frac{1}{D} \frac{d D}{d x}=\frac{d}{d x} \ln D .
$$

Finally, for the $N$-soliton solution of problem (1.1) - (1.4), we obtain the formula

$$
u(x, t)=-2 \frac{d^{2}}{d x^{2}} \ln D .
$$

Thus, we arrive at the following theorem.

Theorem 4.1. Problem (1.1) - (1.4) has multisoliton solutions

$$
u(x, t)=-2 \frac{d^{2}}{d x^{2}} \ln D
$$

where $D$ is defined by formula (4.3) with

$$
\alpha_{n}^{+}(t)=\alpha_{n}^{+}(0) \exp \left\{\int_{0}^{t}\left(8 \chi_{n}^{3} \beta(\tau) u\left(x_{0}, \tau\right)+2 \chi_{n} \gamma(\tau) u\left(x_{1}, \tau\right)\right) d \tau\right\}, \quad n=\overline{1, N},
$$

and $\left(\chi_{j}, \alpha_{j}^{+}(0)\right)$ are the scattering data for the Sturm-Liouville operator with a reflectionless potential $u_{0}(x)$. 
Example 4.1. Consider the following problem

$$
\begin{gathered}
u_{t}+\beta(t) u(0, t)\left(u_{x x x}-6 u u_{x}\right)+\gamma(t) u(1, t) u_{x}=0, \\
u(x, 0)=-\frac{6}{\operatorname{ch}^{2} x}, \quad x \in \mathbb{R}
\end{gathered}
$$

where

$$
\begin{gathered}
\beta(t)=-\left(\sqrt{t^{2}+1}+1\right)(\operatorname{ch}(72 t+78 a(t))+9 \operatorname{ch}(56 t+58 a(t)) \\
+12 \operatorname{ch}(36 t+39 a(t)) \operatorname{ch}(28 t+29 a(t))+10) \\
\times\left(24 \sqrt{t^{2}+1}\left(8 \operatorname{ch}^{2}(4 t+5 a(t))+2 \operatorname{sh}^{2}(32 t+34 a(t))\right)\right)^{-1}, \\
\gamma(t)=-(\operatorname{ch}(72 t+78 a(t)-6)+9 \operatorname{ch}(56 t+58 a(t)-2) \\
+12 \operatorname{ch}(36 t+39 a(t)-3) \operatorname{ch}(28 t+29 a(t)-1)+10) \\
\times\left(24 \sqrt{t^{2}+1}\left(8 \operatorname{ch}^{2}(4 t+5 a(t)-1)+2 \operatorname{sh}^{2}(32 t+34 a(t)-2)\right)\right)^{-1},
\end{gathered}
$$

and $a(t)=\operatorname{arcsh} t$.

In this case, the scattering data of the operator is as follows:

$$
\begin{gathered}
\lambda_{1}(0)=\left(i \chi_{1}(0)\right)^{2}=-1, \quad \lambda_{2}(0)=\left(i \chi_{2}(0)\right)^{2}=-4 ; \\
r^{+}(k, 0)=0, \quad \alpha_{1}^{+}(0)=6, \quad \alpha_{2}^{+}(0)=12 .
\end{gathered}
$$

By Theorem 3.1, we have

$$
\lambda_{1}(t)=-1, \quad \lambda_{2}(t)=-4 ; \quad r^{+}(k, t)=0, \alpha_{1}^{+}(t)=6 e^{\mu_{1}(t)}, \quad \alpha_{2}^{+}(t)=12 e^{\mu_{2}(t)},
$$

where

$$
\begin{aligned}
& \mu_{1}(t)=8 \int_{0}^{t} \beta(\tau) u(0, \tau) d \tau+2 \int_{0}^{t} \gamma(\tau) u(1, \tau) d \tau \\
& \mu_{2}(t)=64 \int_{0}^{t} \beta(\tau) u(0, \tau) d \tau+4 \int_{0}^{t} \gamma(\tau) u(1, \tau) d \tau .
\end{aligned}
$$

Using Theorem 4.1, we find a two-soliton solution to problem (4.5) - (4.6):

$$
u(x, t)=-12 \frac{3+4 \operatorname{ch}(2 x-8 t-10 a(t))+\operatorname{ch}(4 x-64 t-68 a(t))}{(\operatorname{ch}(3 x-36 t-39 a(t))+3 \operatorname{ch}(x-28 t-29 a(t)))^{2}} .
$$

It is known that the solution of the $\mathrm{KdV}$ equation satisfying the initial condition (4.6) looks as follows:

$$
u(x, t)=-12 \frac{3+4 \operatorname{ch}(2 x-8 t)+\operatorname{ch}(4 x-64 t)}{(\operatorname{ch}(3 x-36 t)+3 \operatorname{ch}(x-28 t))^{2}} .
$$

The difference between these two solutions is shown in the following figures: 

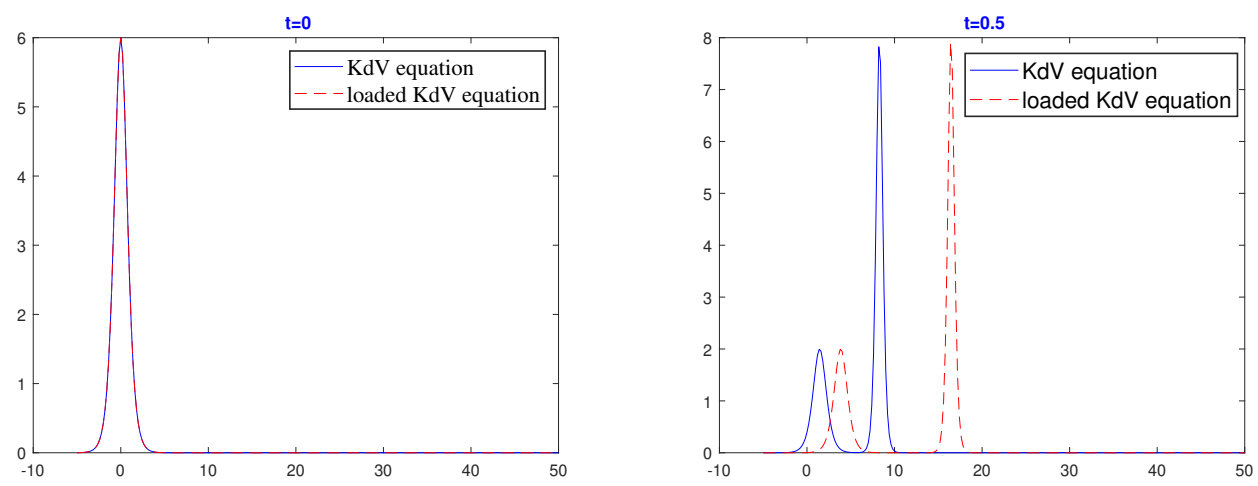

Thus, the following effect has been discovered: in the presence of a loaded coefficient or a term in the $\mathrm{KdV}$ equation, the propagation velocity of a soliton, depending on the coefficients $\beta(t)$ and $\gamma(t)$, will increase or decrease, but the amplitude does not change. However, if a pulse propagates in the form of a soliton of a loaded KdV equation of the form (1.1), then it can transfer information over long distances without distortion and without noticeable loss of intensity than a conventional soliton.

\section{References}

[1] M. Ablowitz, H. Sigur, Solitons and the inverse problem method, Moscow: Mir, 1987.

[2] R. Dodd, J. Eilbeck, J. Gibbon, H. Morris, Solitons and Nonlinear Wave Equations, Moscow: Mir, 1988.

[3] L.D. Faddeev, The Inverse Problem in the Quantum Theory of Scattering, Usp. Mat. Mauk 14:4(88) (1959), $57-119$.

[4] A.S. Fokas, M.J. Ablowitz, Forced Nonlinear Evolution Equations and the Inverse Scattering Transform, Stud. Appl.Math. 80 (1989), 253 - 272.

[5] C.S. Gardner, I.M. Greene, M.D. Kruskal, R.M. Miura, Method for Solving the Korteweg-de Vries Equation, Phys. Rev. Lett. 19 (1967), 1095 - 1097.

[6] C.S. Gardner, G.K. Morikawa, Similarity in the asymptotic behavior of collision-free hydromagnetic waves and water waves, New York: Courant Institute of Mathematical Sciences, New York University, 1960.

[7] I.M. Guseinov, On the continuity of the reflection coefficient of the one-dimensional Schredinger equation, Differential equations, 21:11 (1985), 1993 - 1995.

[8] U.A. Hoitmetov, Integration of the general KdV equation with a self-consistent source in the class of rapidly decreasing complex-valued functions, Reports of the Ac. of Sc. RUz., 5, (2007), $16-20$.

[9] A.B. Khasanov, U.A. Hoitmetov, On integration of Korteweg-de Vries equation in a class of rapidly decreasing complex-valued functions, Russian Math. (Iz. VUZ), 62:3 (2018), $68-78$.

[10] A.B. Khasanov, M.M. Matyakubov, Integration of the nonlinear Korteweg-de Vries equation with an additional term, Theoret. and Math. Phys., 203:2 (2020), 596 607.

[11] A.B. Khasanov, G.U. Urazboyev, On the general Korteweg-de Vries equation with a source in the class of step functions, RAS reports (Doklady RAN), 397:1 (2004), $32-36$.

[12] A.I. Kozhanov, Nonlinear loaded equations and inverse problems, Comput. Math. Math. Phys., 44:4 (2004), $657-675$. 
[13] P.D. Lax, Integrals of Nonlinear Equations of Evolution and Solitary Waves, Comm. Pure and Appl. Math., 21:5 (1968), 467 - 490.

[14] J. Leon, A. Latifi, Solution of an initial-boundary value problem for coupled nonlinear waves, J. Phys. A: Math.Gen. 23 (1990), $1385-1403$.

[15] B.M. Levitan, Inverse Sturm-Liouville Problems, Nauka, Moscow, 1984.

[16] A.A. Lugovtsov, Propagation of Nonlinear Wawes in a Unhomogeneous Gas-Liquid Medium. Derivation of the Wave Equations Close to Korteweg - de Vries, Applied Mech. and tech. Phys., 50:2 (2009), $188-197$.

[17] A.A. Lugovtsov, Propagation of Nonlinear Waves in a Gas-Liquid Medium. Exact and Approximate Analytical Solutions of Wave Equations. Applied Mech. and tech. Phys., 51:1 (2010), $54-61$.

[18] V.A. Marchenko, Sturm-Liouville Operators and Their Applications, Kiev, Naukova Dumka, 1977.

[19] V.K. Mel'nikov, Exact Solutions of the Kortewed-de Vries Equation with a Selfconsistent Source, Phys. Lett. A, 128:9 (1988), 488-492.

[20] A.M. Nakhushev, Loaded equations and their applications, Diff. Urav. 19:1 (1983), $86-94$.

[21] A.M. Nakhushev, Equations of Mathematical Biology, Moscow: Vysshaya Shkola, 1995.

[22] R.Z. Sagdeev, Containment of plasma by the pressure of a standing electromagnetic wave, Plasma physics and the problem of controlled thermonuclear reactions, 4 (1958), $346-362$.

[23] L.A. Takhtadzhyan, L.D. Faddeev, Hameltonian approach in the theory of solitons, Moscow: Nauka, 1986.

[24] G.U. Urazboyev, A.B. Khasanov, Integrating the Korteweg-de Vries Equation with a Self-Consistent Source and "Steplike" Initial Data, Theoret. and Math. Phys., 129:1 (2001), 1341 - 1356.

[25] A.B. Yakhshimuratov, M.M. Matyokubov, Integration of loaded Korteweg-de Vries equation in a class of periodic functions, Russian Math. (Iz. VUZ), 60:2 (2016), 72 -76 .

[26] V.A. Yurko, Introduction to the theory of inverse spectral problems, Moscow: Fizmatlit, 2007.

[27] V.E. Zakharov, S.V. Manakov, S.P. Novikov, L.P. Pitaevsky, The theory of solitons. The Inverse Problem Method, Moscow: Nauka, 1980.

Aknazar B. Hasanov

Samarkand State University, 15 University Boulevard, Samarkand, 140104, Uzbekistan

E-mail address: ahasanov2002@mail.ru

Umid A. Hoitmetov

Khorezm Branch of the Romanovsky Institute of Mathematics and Urgench State University, 14 H.Alimjan, Urgench, 220100, Uzbekistan

E-mail address: x_umid@mail.ru

Received: January 26, 2020; Accepted: June 1, 2021 